\title{
Numerical Results of Modeling Semiconductor Sensor Layers in SAW Gas Sensors
}

\author{
T. HEJCZYK ${ }^{a}$, M. URBAŃCZYK ${ }^{b}$ AND W.P. JAKUBIK ${ }^{c}$ \\ ${ }^{a}$ ENTE Sp. z o.o., Gaudiego 7, 44-100 Gliwice, Poland \\ ${ }^{b}$ Faculty of Electrical Engineering, Silesian University of Technology, Krzywoustego 2, 44-100 Gliwice, Poland \\ ${ }^{c}$ Institute of Physics, Silesian University of Technology, Krzywoustego 2, 44-100 Gliwice, Poland \\ The paper presents the numerical results of investigations of the layered gas surface acoustic waves sensor. \\ The base electric load of the piezoelectric acoustic line is predicted by the effect of surface acoustic waves velocity \\ changes vs. surface conductivity, which depends on the profile concentration by gas diffused molecules into the \\ porous film. Inside the sensor layer Knudsen's model of gas diffusion was used.
}

PACS numbers: 77.65.Dq, 68.35.Iv, 07.07.Df

\section{Introduction}

The paper summarizes the acoustoelectric theory [1], i.e. the Ingebrigtsen formula [2], the impedance transformation law, gas concentration profiles, and predicts the influence of a thin semiconductor sensor layer in the Knudsen gas diffusion model on the velocity of the surface acoustic waves (SAW) in a piezoelectric acoustic waveguide [3]. The paper proposed a new theory of analyzing a SAW gas sensor. As an example of the adaptability of this theory numerical results are presented in a thin film of a semiconducting layer placed on a $Y-Z \mathrm{LiNbO}_{3}$ SAW waveguide $[4,5]$, showing the changes in velocity of propagation, depending on the concentration of the surrounding hydrogen gas, the thickness of the semiconductor, the pore radius and the temperature exposure to gas.

\section{Numerical modeling}

According to the transformation law we calculated the common admittance at the surface $y=0$. The gas concentration profile was counted basing on concentration of the profiles [6], which are different for gases, depending from the Knudsen diffusion constants [7], temperature, molecular weight, the gas constants $R$ and the thickness of the porous sensing materials with an assumed porous radius. A simple model of a structure made of porous sensing material is shown in Fig. 1. Molecular hydrogen dissociated to atomic hydrogen on the outer catalytic surface. Adsorbed hydrogen atoms then act as electrical dipoles at the metal-semiconductor interface and create changes in the work function in the surface conductivity of the semiconductor material $[8,9]$. The target gas, diffusing in the sensing layer, is consumed gradually in the

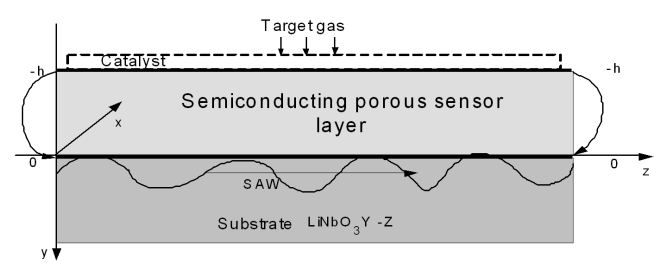

Fig. 1. Actual model of a semiconducting gas sensor layer.

surface reaction. Under steady-state conditions the gas concentration inside the sensing layer decreases with the increasing diffusion depth, resulting in a gas concentration profile which depends on the rates of the surface reaction and diffusion reaction. Other gases like $\mathrm{CO}, \mathrm{CO}_{2}$, $\mathrm{NO}_{2}$ can diffuse likewise hydrogen into porous sensing material depending on its temperature, molecular weight and the physical parameters of the sensing layers. The main assumption was that it is Knudsen's diffusion and that the semiconductor layer is a stack of infinitesimally thin sheets whose conductance is given by $\sigma(y)$, where $y$ is the distance from the piezoelectric substrate.

Gas concentration inside a sensor layer is not constant. It decreases with the depth due to the surface reaction and diffusion. Then we assumed that the film is a uniform stack of infinitesimally thin sheets with variable gas molecule concentrations.

A main assumption is that the sheet electrical conductance $\sigma$ exposed to the target gas is linear in the gas concentration $C_{\mathrm{A}}[1]$ :

$$
\sigma(y)=\sigma_{0}\left[1+a C_{\mathrm{A}}(y)\right] .
$$

Numerical analyses were performed out in the Python environment. We use equation (29) in [1]: 


$$
\begin{aligned}
& \frac{\Delta v}{v_{0}}=-\operatorname{Re}\left(\frac{\Delta k}{k_{0}}\right)=-\frac{K^{2}}{2} \sigma_{T_{2}}^{2}\left(1+a C_{\mathrm{A}}\right)^{2} \\
& \times\left[1+\sum_{i=1}^{n-1} f\left(y_{i}, \sigma\left(y_{i}\right)\right)\right]^{2} \\
& /\left\{\sigma_{T_{2}}^{2}\left(1+a C_{\mathrm{A}}\right)^{2}\left[1+\sum_{i=1}^{n-1} f\left(y_{i}, \sigma_{T_{2}}\right)\right]^{2}\right. \\
& \left.\quad+\left[1+\sum_{i=1}^{n-1} g\left(y_{i}, \sigma\left(y_{i}\right)\right)\right]^{2}\left(v_{0} C_{\mathrm{S}}\right)^{2}\right\}
\end{aligned}
$$

and

$$
\sigma_{T_{2}}=\sigma_{T_{1}} \exp \left(\frac{E_{\mathrm{g}}}{2 k} \frac{T_{2}-T_{1}}{T_{1} T_{2}}\right),
$$

where $T_{1}=300 \mathrm{~K}, \sigma_{T_{1}}=\sigma_{T_{1}}(300 \mathrm{~K})=\sigma_{0}=\sigma_{\mathrm{S}}$ in the program, depending on the following values: gas concentration, layer thickness, sensor temperature and radius of the pores [3].

\section{Numerical results}

Figure 2 illustrates the dependence of the Rayleigh wave velocity on the concentration of $\mathrm{H}_{2}, \mathrm{CO}_{2}, \mathrm{NO}_{2}$, $\mathrm{NH}_{3}$. The correlation is depicted by assuming the following values for the involved constant: $\sigma_{\mathrm{s}}=v_{0} C_{\mathrm{S}}=$ $1.6 \times 10^{-8} \Omega^{-1}$, sensitivity coefficient $a=1 \mathrm{ppm}^{-1}$, thickness of the sensor layer $100 \mathrm{~nm}$, temperature $300 \mathrm{~K}$, pore radius $5 \mathrm{~nm}$.

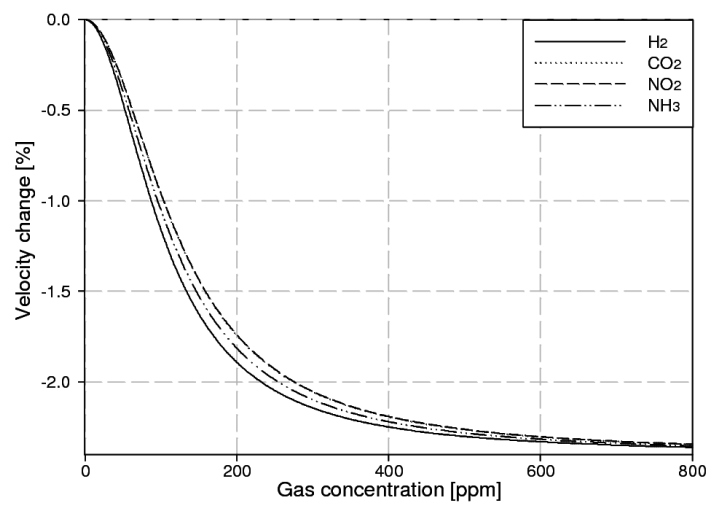

Fig. 2. Velocity changes of the SAW wave propagation vs. gas concentration around the sensor layer for $\mathrm{H}_{2}$, $\mathrm{CO}_{2}, \mathrm{NO}_{2}, \mathrm{NH}_{3}$.

Figure 3a,b illustrates the SAW velocity dependence on the thickness of the sensor layer for $\mathrm{H}_{2}, \mathrm{CO}_{2}, \mathrm{NO}_{2}, \mathrm{NH}_{3}$. The correlation is depicted by assuming the following values for the constant: $\sigma_{\mathrm{s}}=v_{0} C_{\mathrm{S}}=1.6 \times 10^{-8} \Omega^{-1}$ (start point of work sensor), for the thickness $h=100 \mathrm{~nm}$, sensitivity coefficient $a=1 \mathrm{ppm}^{-1}$, gas concentration $300 \mathrm{ppm}$, temperature $320 \mathrm{~K}$, pore radius $2 \mathrm{~nm}$.

Figure 4 illustrates the SAW velocity dependence on the temperature of the sensor layer for $\mathrm{H}_{2}, \mathrm{CO}_{2}, \mathrm{NO}_{2}$, $\mathrm{NH}_{3}$. The correlation is depicted by assuming the following values for the involved constant: $\sigma_{\mathrm{s}}=v_{0} C_{\mathrm{S}}=$ $1.6 \times 10^{-8} \Omega^{-1}$, sensitivity coefficient $a=1 \mathrm{ppm}^{-1}$,

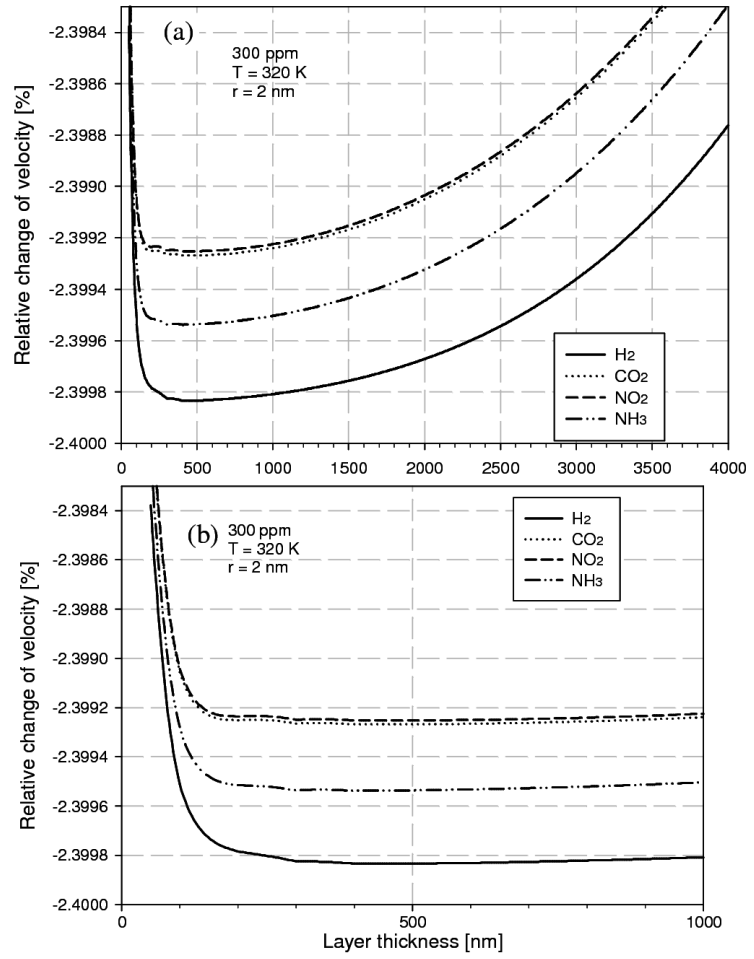

Fig. 3. SAW wave propagation velocity changes vs. thickness of the sensor layer for $300 \mathrm{ppm} \mathrm{H}_{2}, \mathrm{CO}_{2}, \mathrm{NO}_{2}$ or $\mathrm{NH}_{3}$. Pore radius $r=2 \mathrm{~nm}, T=320 \mathrm{~K}$.

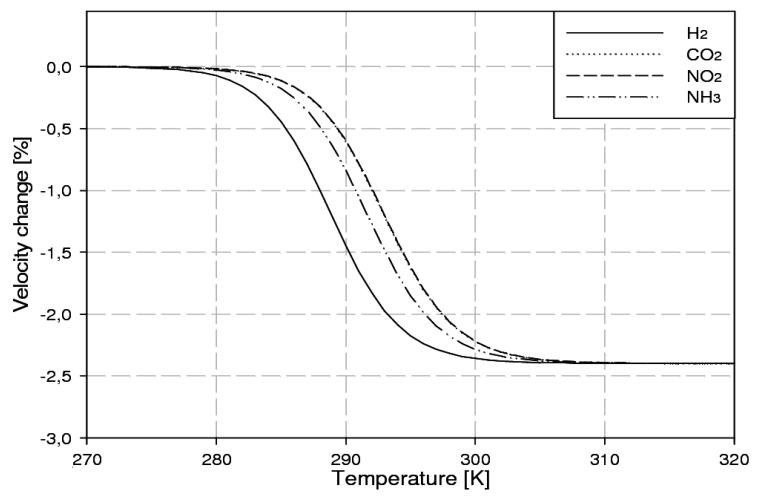

Fig. 4. SAW wave propagation velocity changes vs. temperature of the sensor layer for $\mathrm{H}_{2}, \mathrm{CO}_{2}, \mathrm{NO}_{2}, \mathrm{NH}_{3}$ gases.

thickness of the sensor layer $100 \mathrm{~nm}$, gas concentration $300 \mathrm{ppm}$, pore radius $5 \mathrm{~nm}$.

Figure 5 illustrates the SAW velocity dependence on the pore radius of the sensor layer for $\mathrm{H}_{2}, \mathrm{CO}_{2}, \mathrm{NO}_{2}$, $\mathrm{NH}_{3}$. The correlation is depicted by assuming the following values for the involved constant: $\sigma_{\mathrm{s}}=v_{0} C_{\mathrm{S}}=$ $1.6 \times 10^{-8} \Omega^{-1}$, sensitivity coefficient $a=1 \mathrm{ppm}^{-1}$, thickness of the sensor layer $100 \mathrm{~nm}$, gas concentration 300 ppm, temperature $300 \mathrm{~K}$.

Figure 6 illustrates the SAW velocity dependence on the temperature of the sensor layer for $\mathrm{H}_{2}$ and different 


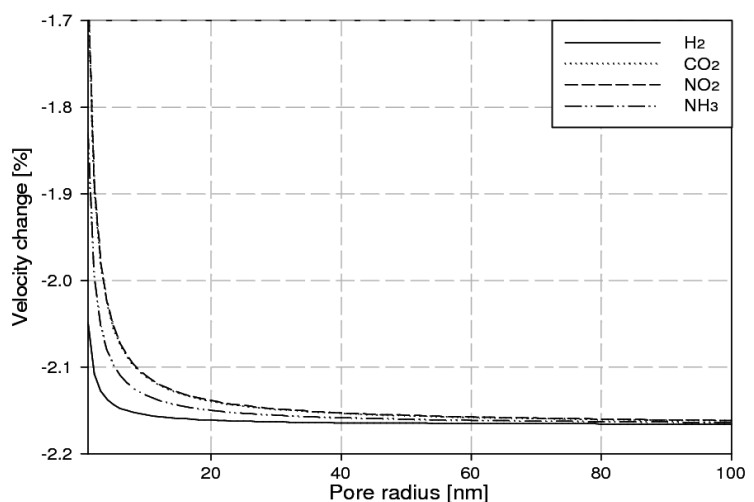

Fig. 5. SAW wave propagation velocity changes vs. pore radius of the sensor layer for $\mathrm{H}_{2}, \mathrm{CO}_{2}, \mathrm{NO}_{2}, \mathrm{NH}_{3}$.

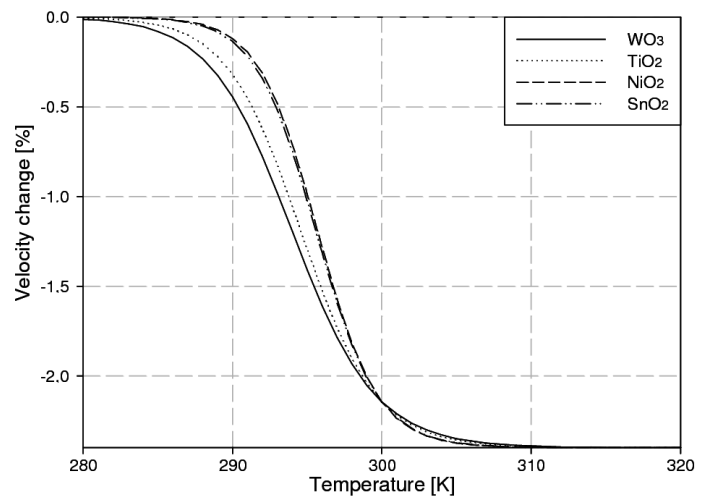

Fig. 6. SAW wave propagation velocity changes vs. temperature of the sensor layer of $\mathrm{WO}_{3}, \mathrm{TiO}_{2}, \mathrm{NiO}$, $\mathrm{SnO}_{2}$ for $\mathrm{H}_{2}$ gas.

layers of the type $\mathrm{WO}_{3}, \mathrm{TiO}_{2}, \mathrm{NiO}, \mathrm{SnO}_{2}$. The correlation is depicted by assuming the following values for the involved constant: $\sigma_{\mathrm{s}}=v_{0} C_{\mathrm{S}}=1.6 \times 10^{-8} \Omega^{-1}$, sensitivity coefficient $a=1 \mathrm{ppm}^{-1}$, thickness of the sensor layer $100 \mathrm{~nm}$, gas concentration for $\mathrm{H}_{2}, 300 \mathrm{ppm}$, pore radius $5 \mathrm{~nm}, E_{\mathrm{g}}=2.7 \mathrm{eV}$ for $\mathrm{WO}_{3}, E_{\mathrm{g}}=3 \mathrm{eV}$ for $\mathrm{TiO}_{2}$, $E_{\mathrm{g}}=3.8 \mathrm{eV}$ for $\mathrm{NiO}, E_{\mathrm{g}}=3.7 \mathrm{eV}$ for $\mathrm{SnO}_{2}$ as for the solid material [8].

\section{Conclusions}

a) In this paper numerical results have been shown for gases $\mathrm{H}_{2}, \mathrm{CO}_{2}, \mathrm{NO}_{2}, \mathrm{NH}_{3}$ and solid sensing layers like $\mathrm{WO}_{3}, \mathrm{TiO}_{2}, \mathrm{NiO}, \mathrm{SnO}_{2}$. b) We can use the profile of the concentration gas in the layer in order to point response of the gas sensor.

c) We divided the porous semiconductor layer into sublayers. According to the transformation law we calculated the influence of the impedance above the piezoelectric substrate on the relative change of velocity versus concentration, thickness, size of the pores, temperature.

d) We assumed the Knudsen diffusion in porous sensing materials in which pores in range from 1 to $100 \mathrm{~nm}$ in radius do prevail.

e) In the analytical model we introduce sheet conductance which is assumed to be linear in the gas concentration.

f) The designed mathematic model of SAW sensor is based on the electric effect which results from changes in the electric surface conductivity of the layers $\sigma_{\mathrm{s}}(y)$, due to the interaction of this chemically active layer with gas.

g) We are going to optimize and to modeling the construction of the SAW sensor in the future.

\section{Acknowledgments}

This work is financed as the grant of Ministry of Science and Higher Education No. N N505 374237.

\section{References}

[1] T. Hejczyk, M. Urbańczyk, W. Jakubik, Acta Phys. Pol. A 118, 1148 (2010).

[2] B.A. Auld, Acoustic Fields and Waves, Vol. 2, Wiley, New York 1973.

[3] G. Sakai, N. Matsunaga, E. Shimanoe, N. Yamazone, Sensors Actuators B 80, 125 (2001).

[4] B. Pustelny, T. Pustelny, Acta Phys. Pol. A 116, 383 (2009).

[5] T. Hejczyk, M. Urbańczyk, W. Jakubik, Acta Phys. Pol. A 118, 1152 (2010).

[6] J.W. Gardner, Sensors Actuators B 1, 166 (1990).

[7] T. Pustelny, A. Opilski, B. Pustelny, Acta Phys. Pol. A 114, A-181 (2009).

[8] T. Pisarkiewicz, Gas Microsensors, AGH, Kraków 2007.

[9] J. Portier, H.S. Hilal, Prog. Solid State Chem. 32 207 (2004). 